\title{
Mapping of Force: a Process of Parametric Design from the Prototype Mechanical Mechanism to the Structural Form of a Woven HYPAR Shell
}

\author{
Kang Qiang Lin ${ }^{1, a}$, Chao Hao Su, b \\ ${ }^{1,2}$ Architecture Design and Research Institute, South China University of Technology, Guangzhou, 510640, China
}

\begin{abstract}
From a new perspective of structural engineering, with the integration of the 1st and 3rd principal stress distribution of HYPAR shell structure into the selection of texture variation zone, this paper analyzes and demonstrates the key questions of how to establish the association between the force-flow, the texture direction and the form parameters; how to develop a dynamic, performance-optimized and adaptive iteration model of HYPAR shell structural form by setting and adjusting structurally sensitive parameters; and how to realize the process of parametric design from the mechanical mechanism of the prototype to the structural form of the woven HYPAR shell.
\end{abstract}

\section{Introduction}

In architectural parametric design, a new interdisciplinary research question has emerged as how to effectively coordinate and reconstruct the relationships between stress and form ${ }^{[1,2]}$ for achieving the unification of free forms and objective constraints. To express the inner logic of structural mechanics in the form of an architectural language has created an important branch of modern architecture, that is, structure as architecture ${ }^{[3]}$. However, with the arrival of the era of digital architectural design, the "emergence" ${ }^{[4]}$ of complex forms created by complex mathematical algorithms and complicated computer programs has brought severe challenges and posed a novel research topic for the integration and expression of structural mechanics. Schumacher ${ }^{[5,6]}$. has recently declared "trying more and more to move away from the freeform play with complex curvature towards the disciplined use of structural form-finding algorithms " ${ }^{[7]}$.

From yet another perspective, the mechanical prototype of a structure can be adopted as the starting point to analyze its fundamental stress mechanism and inherent relationship, and digital tools can be introduced to realize the adaptive topology of geometric forms. This paper further discusses how to establish the correlations between the mechanical mechanisms, such as stress distribution and force flow ${ }^{[8]}$, and the digital geometric forms. It also demonstrates the parametric design process of the form topology of HYPAR shell structures based on the form-innovation and optimization tests of the woven texture pattern of a response mechanics mechanism.

\section{Mechanical mechanism and a misunderstanding}

Basis exploration: Structures play a role in form resistance, and define the relationships between stress and form, which are not so intuitive. Thus, the mechanical mechanism of a structural prototype serves as the prerequisite of the mapping of force onto form. Two mechanical logics related to the form resistance and parametric design must be clarified at this stage, they are, stress distribution and force flow.

Not merely a stress nephogram: Generally the architectural parametric design only employs the nephogram that reflects stress distribution, but has neglected force flow trace - another important factor that is fixed but hidden. Thus, there is a common misunderstanding in the integration of mechanics obtained by architectural parametric design. Consequently, a one-sided reflection of stress distribution is employed as a substitute for the reflection of the mechanical mechanism. The misunderstanding is potentially erroneous as force flow plays a very important and special role, especially in the HYPAR shells. Geometrically, a HYPAR shell is generated by revolving a straight line along another orthogonal bus. Doing this may easily lead to a mechanical misunderstanding, i.e., the force is transferred to the boundary along the orthogonal straight line direction. However, the laws of physics do not work in this way: he force transfer always searches for the most favorable form resistance path, that is, there is a superposition of suspension cable mechanism and an arch force transfer mechanism on the two diagonal directions, respectively ${ }^{[9]}$ (see Fig.1).

Difference between the mathematical and physical significance: To obtain the unit form of a parametric design with an effective overall form resistance action, adapting to the path of cable plus arch seems to be both necessary and critical. Conversely, damaging this potential mechanical logic would make it impossible to construct a shell structure. Rather what would be constructed would be a shell-shaped geometric body having only a purely mathematical significance, as the force transfer mechanism would be changed.

\section{Force flow and priority of mapping directivity}

For brittle materials made of reinforced concrete, in line with the first strength theory(max normal stress theory), the first principal stress plays a dominant role. Therefore, the suspension cable mechanism will have a priority over the arch mechanism. Based on the mapping forms of the two forces, we can obtain a distinct data comparison through mechanical calculation (see Fig.2).

1) Arch mechanism as the priority: On the one

alinkangqiang0101@126.com, bsuchaohao@scut.edu.cn

This is an Open Access article distributed under the terms of the Creative Commons Attribution License 4.0, which permits unrestricted use distribution, and reproduction in any medium, provided the original work is properly cited. 

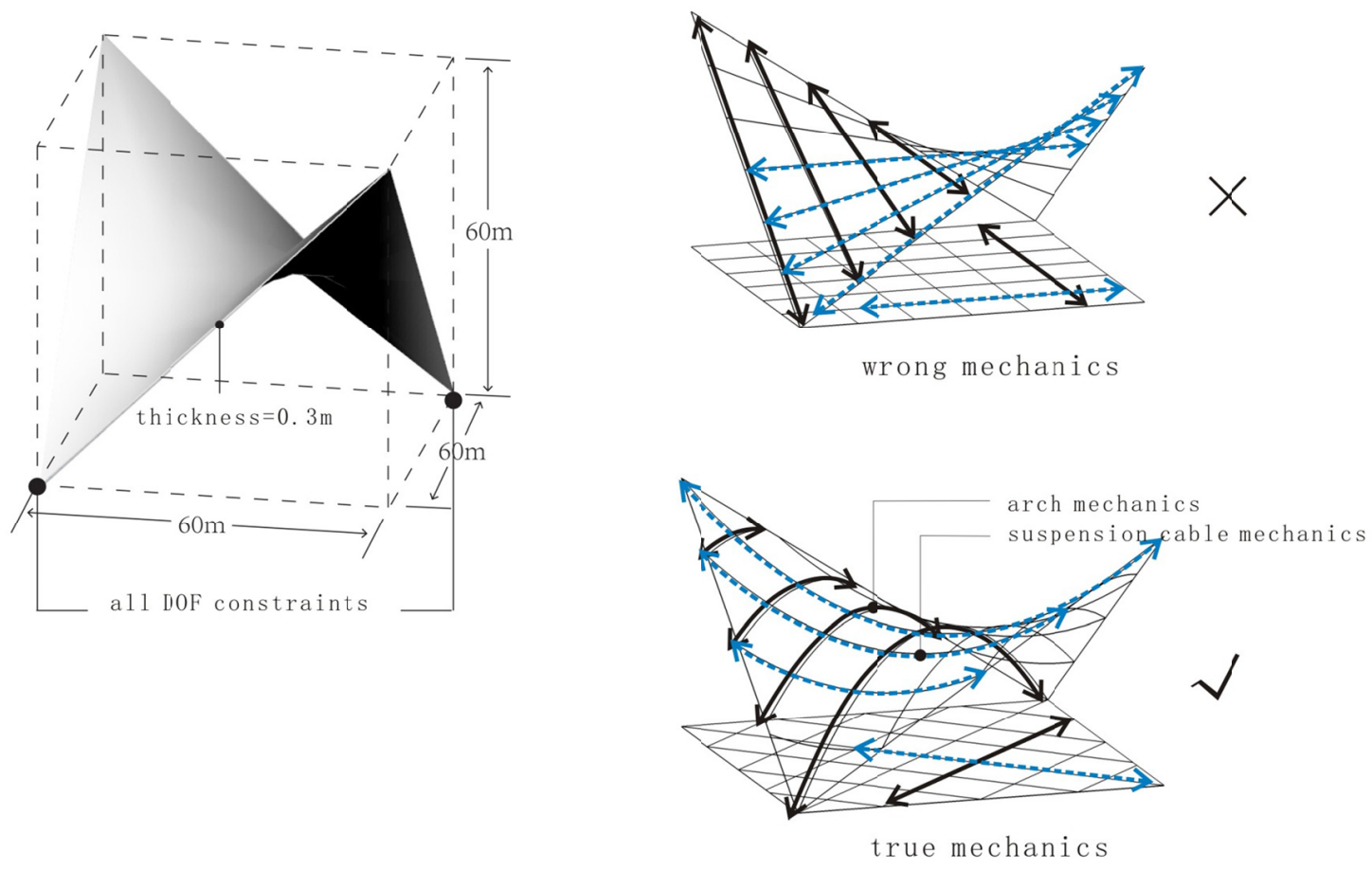

Fig. 1. Analysis of structural mechanics

hand, the tensile stress fails to be transferred along the diagonal direction. This causes the tensile stress to exceed $20 \mathrm{MPa}$ at stress concentrated zones, thus necessitating a high reinforcement ratio and subsequently causing difficulty in reinforcement. On the other hand, due to the lack of a structural member in the tensile direction of the

suspension cable, the overall rigidity is discounted, and the large deformation on the tensile end fails to be satisfactorily controlled (deformation value having reached as high as 1.124);

2) Suspension cable mechanism as the priority: In the second circumstance, with the directional adjustment of the mapping unit, the dominant tensile direction is cleared, the tensile stress remains fairly uniform within $13 \mathrm{MPa}$. The deformation value has recovered to 0.858 , and the overall deformation is relatively uniform without any sudden variation. Although the pressure transfer path is slightly tortuous, it has not incurred any change in the prototype mechanical mechanism.

\section{Iteration of the mechanical form}

Following the established mechanical mechanism, the degree of spatial freedom of the structural form has a flexible range that can adapt to the architecturally significant demands such as light and shadow, air flow, thermal environment and visual interface dynamics. Thus, an iteration model can be generated between spatial form and mechanical performance by selecting or combining structurally sensitive parameters with relevant evaluation and analysis.

\subsection{Structural calculation and sensitive parameters}

The conventional structural mechanical calculation is usually divided into two steps: first, some fixed unit models are set to simplify complex forms; and second, the calculation results are employed for the detailed checking of the specific forms of local zones. Apparently, form parameter information, or even more sensitive parameters, have been dleleted in the name of simplification. Firstly, it will result in a major error in the mechanical analysis when compared to a practical situation. Secondly, the process of adopting sensitive parameters for a dynamic regulation is suspended. Thus, as far as the woven structural form is concerned, the conventional simplified mechanical calculation has serious limitations, and hence, the structural calculation of the holo-information model seems to be more applicable and important.

The logic generated on the basis of the woven texture of the force transfer mechanism is as shown in Fig.3. Holing and warping are, respectively, conducted on the upper and lower layers, and are mutually superposed and alternated. Thus, the radius of the circle produces a correspondence with the first principal stress nephogram within its numerical interval. Accordingly it 

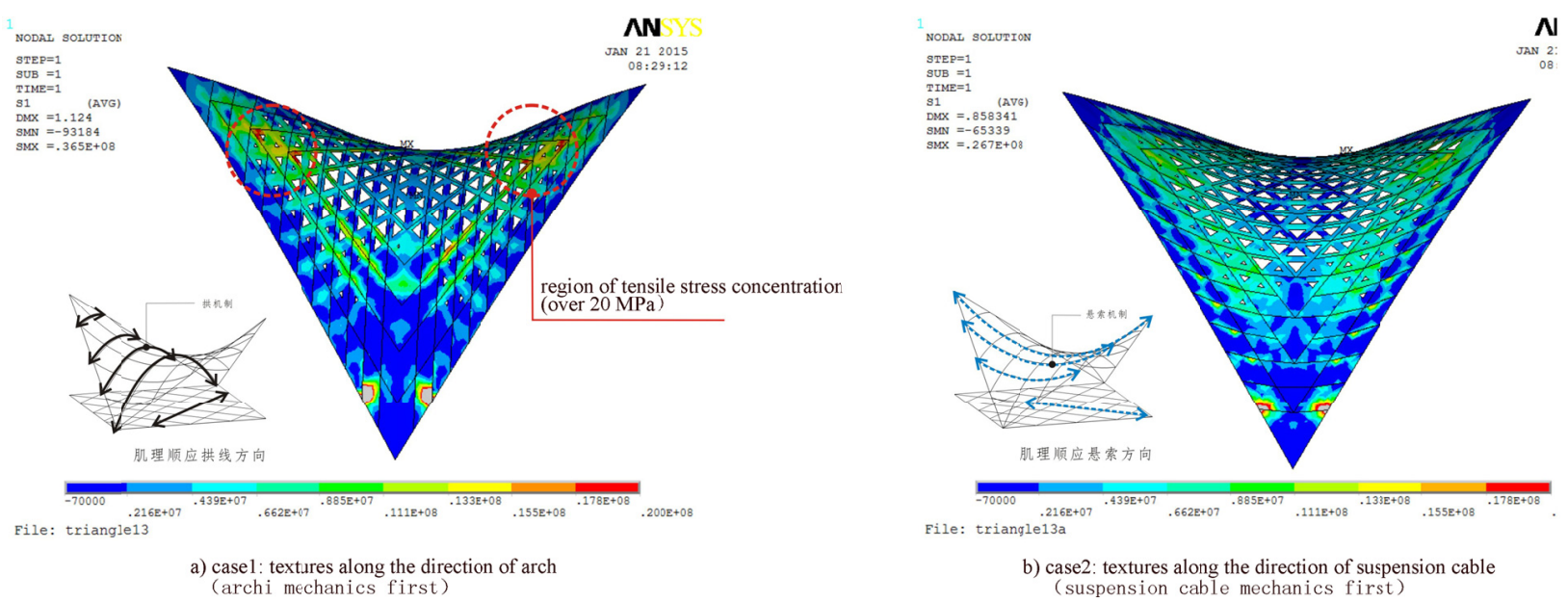

Fig. 2. Test of mechanical performance between two mapping ways according to arch mechanics and suspension cable mecha

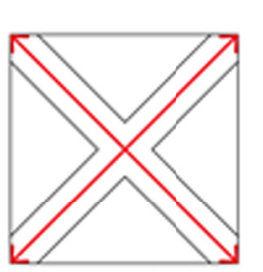

a) stress paths of arch $\&$ suspension cable direction

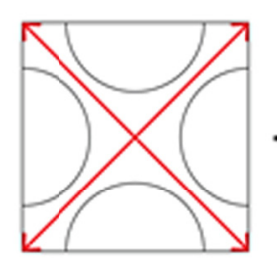

b) layer 1:circular holes near four edges

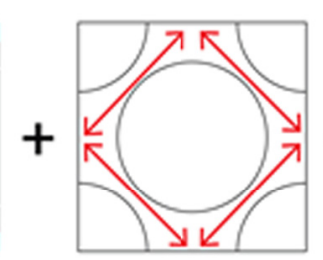

c) layer 2 :circular holes at corners and center

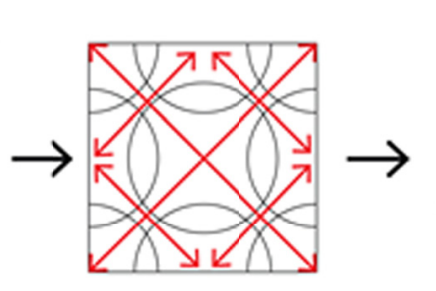

d) superposed fold of two layers
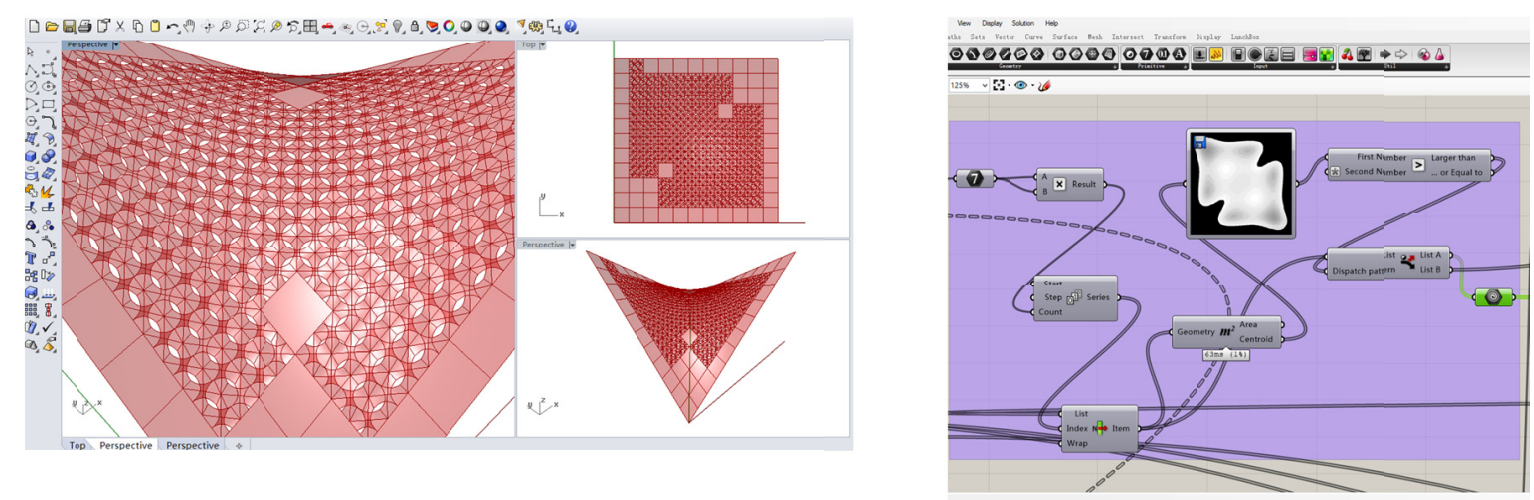

f) a woven HYPAR shell generated with Grasshopper responding to arch and suspension cable mechanics

Fig.3. Woven texture generation and its parametric design process based on prototype structural mechanics(arch + suspension

cable)

reflects and regulates the distribution of the first principal stress as the first structural sensitive parameter.

The relative scale of warping not only realizes the woven form of the texture but also reflects the degree of interference with the prototype mechanical mechanism

This study conducted the iteration tests by three number scales and three holing rates (the holing rate $\mu$ is defined as the ratio of the round hole diameter $d$ to the unit's side length $L^{\prime}$, i.e., $\mu=d / L^{\prime}$ '). See the results in Fig.4.

1) Small-number scale: The force transfer path of suspension cable plus arch can be determined from the principal stress vector diagram. When the holing rate $\mu=0.85,0.78$, there is a marked concentration of stress in the tensile zone (exceeding $20 \mathrm{MPa}$ ); when $\mu=0.70$, by having warping as the second structural sensitive parameter.

\subsection{Iteration model between unit number and holing} rate

stress concentration is eliminated and the peak displacement DMX declines from 0.734 to 0.664 ;

2) Medium-number scale: The principal stress vector diagram shows, relatively clearly, the force transfer path of suspension cable plus arch. When $\mu=0.85$, there is a local concentration of stress on the edges of the round circle in the tensile zone; when $\mu=0.78$, stress concentration gradually disappears, and declines to a level below $30 \mathrm{MPa}$; when $\mu=0.70$, stress distribution is uniform, and the peak displacement DMX declines from 0.839 to 0.683 ; 

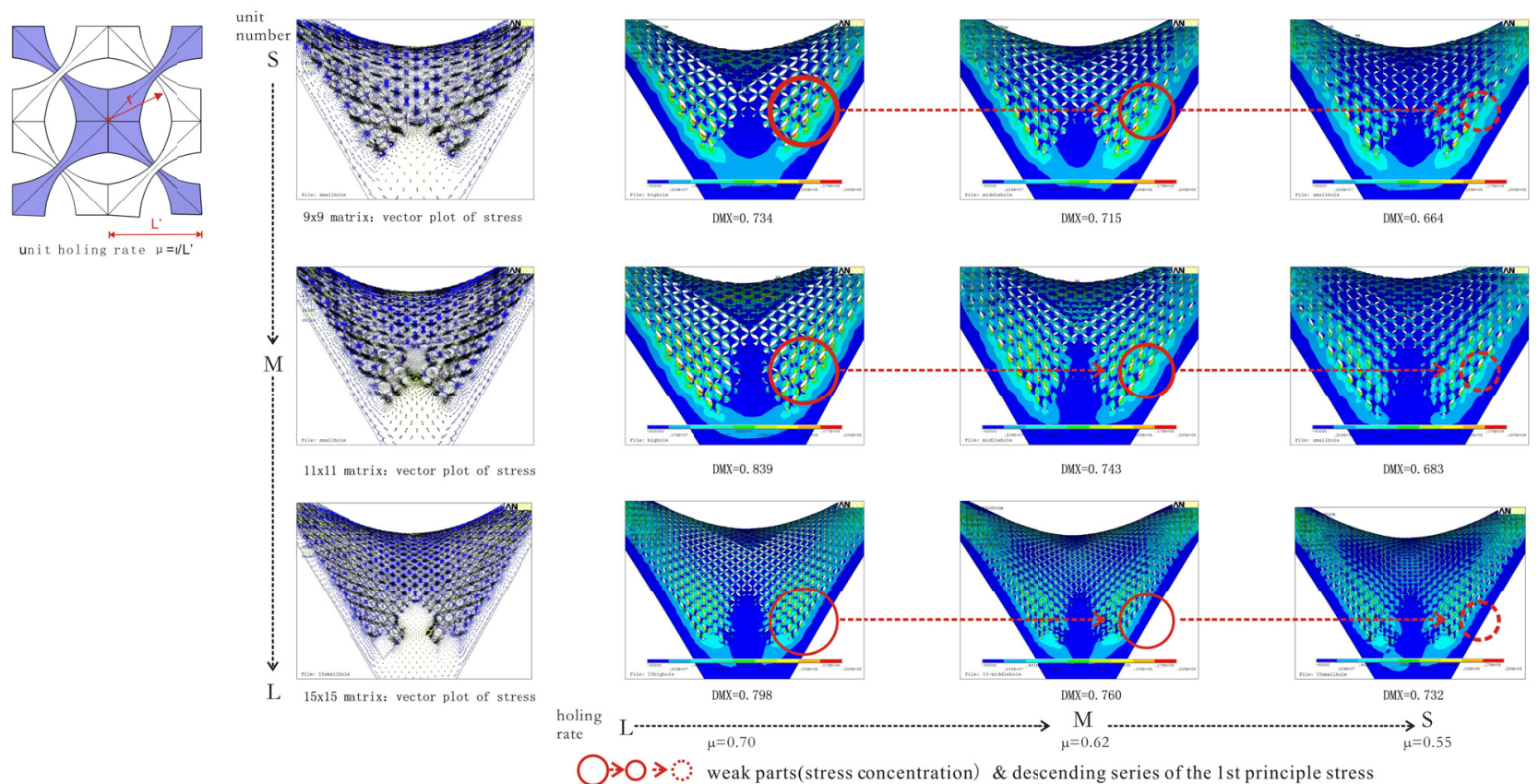

Fig.4. Structural performance in the iteration process of unit number and holing rate

3) Large-number scale: The principal stress vector diagram clearly shows the force transfer path of suspension cable plus arch. Stress distribution is uniform under the three holing rates, and there is no stress concentration zone with stress exceeding $20 \mathrm{MPa}$.

To sum up, the prototype mechanical mechanism of the shell is presented with the gradual increase in unit number. The holing rate has a relatively high structural sensitivity when the unit number is relatively small, and presents a trend of declining degree of interference with a gradual increase in unit number. When the unit number becomes relatively large, the interference of the holing rate is diminished. With a gradual increase in unit number, the peak displacement DMX shows a generally increasing trend but the overall rigidity shows a gradually decreasing trend. However, the reduction in overall rigidity can be compensated by adjusting the holing rate.

\subsection{Spatial creation of mechanical form}

After a series of deduction, simulation and experimental demonstration linked to the structural performance, a new combined model has been proposed for potential stress distribution, force flow trace and external building spatial forms. The performance-based structural form of the shell has provided the basis and theme for the creation of an architectural visual space. On the one hand, there exists a concentration of force potential, i.e., the counter-gravity form of shape. Considering the structural stability, the supporting area of the landing end can be appropriately enlarged to form a triangular support (when combined with the other landing points), in which case the other two suspended end points will construct a large cantilever resembling two flying wings and presenting a counter-gravity visual tension. Moreover, there is the flow of stress, i.e., the woven texture. The woven texture has essentially overturned the all-solid surface, enclosed the spatial form of the shell, and introduced the natural elements while generating a new interface form, thus creating a light and shadow-alternated spatial effect. The diversified spatial form realized through scheduling sensitive parameters can better adapt to the building functions. As far as spatial creation is concerned, the light and shadow effect and force flow rhythms, offered

by the variation in the structural performance-based texture form, present a visual motivation that is not possible in the traditional enclosed shells .

\section{Conclusions}

A building can not change its forms randomly like sculptures, and a structure does not necessarily mean a restraint without freedom. The development of geometric forms under parametric design technology must find a new point of combination with the logic of structural mechanics. an effective technical route was proposed to unify force-driven shaping and resistance through forms, starting with a structural prototype with its inner mechanical mechanism analyzed and, then, utilized to reflect the physical law onto the geometric form. The route is also an important way of realizing the permanent rational controllability of the generation of complex forms during the whole parametric design process. As an example, a woven shell structure was discussed as a quantitative dlemonstration guided by the creation idea and the experimental route of "mapping of force". The key lies in how to analyze the mechanical mechanism of the structural prototype and understanding 
and utilizing the force flow to avoid intuitive misunderstanding. Extension of the proposed technical route will be worthwhile to further explore and forecast the mechanical mapping of more structural prototypes.

\section{Acknowledgment}

The research described in this paper is supported by National Natural Science Foundation of China ( 51308218), Pearl River S\&T Nova Program of Guangzhou(201506010038) and "the Fundamental Research Funds for the Central Universities" ( 2014ZM0012). The corresponding author is Chaohao $\mathrm{Su}$.

\section{References}

[1]. Wanda J. Lewis. Modeling of Fabric Structures and Associated Design Issues [J]. Journal of Architectural Engineering, June, 2013,vol.19:81-88.

[2]. Edward Allen, Waclaw Zalewski. Form and Forces: Designing Efficient, Expressive Structures[M]. John Wiley and Sons Ltd, October 23, 2009.

[3]. Andrew Charleson. Structure As Architecture: A
Source Book for Architects and Structural Engineers[M]. Routledge(2nd Revised edition). July $1,2014$.

[4]. Michael Weinstock. The Architecture of Emergence: The Evolution of Form in Nature and Civilisation[M]. Wiley, April 6, 2010.

[5]. Patrik Schumacher. The Autopoiesis of Architecture: A New Framework for Architecture[M]. Wiley(1 edition), January 18, 2011.

[6]. Patrik Schumacher. The Autopoiesis of Architecture, Volume II: A New Agenda for Architecture[M] . Wiley(1 edition), May 7, 2012.

[7]. Yang Yang, Cristopher D. Moen, James K. Guest. Three-Dimensional Force Flow Paths and Reinforcement Design in Concrete via Stress-Dependent Truss-Continuum Topology Optimization[J]. Journal of Engineering Mechanics, January 2015,vol.141: 04014106-1-10

[8]. Heino Engel, Ralph Rapson. Structure Systems[M]. Hatje Cantz, October, 2006.

[9]. AliReza Habibi. New Approximation Method for Structural Optimization[J]. Journal of Computing in Civil Engineering, 2012, vol.26:236-247. 